Revista de Filosofía

ISSN: 0034-8244

https://dx.doi.org/10.5209/resf.65054

\title{
Fronteras táctiles. Perspectivas en torno a la mano y el tacto en elaboraciones de Husserl, Heidegger y Derrida
}

Luis Fernando Butierrez ${ }^{1}$

Recibido: 10 de julio de 2019 / Aceptado: 26 de junio de 2020

Resumen. En el presente artículo proponemos un abordaje de los análisis en torno al tacto y la mano en trabajos fundamentales de Husserl y Heidegger, en un diálogo con el análisis respectivos de J. Derrida. Por la vía de una lectura que reconoce continuidades y despliegues, buscaremos demostrar que las elaboraciones prácticas del tocar desarrolladas por Derrida articulan una comprensión en cierta continuidad con aquellas elaboraciones tradicionales, en el marco de una lectura singular de los textos respectivos.

Palabras clave: tacto; mano; Husserl; Heidegger; Derrida.

\section{[en] Tactile borders. Perspectives on hand and touch in elaborations by Husserl, Heidegger and Derrida}

Abstract. In the present article we propose an approach to the analysis around the touch and the hand in fundamental works of Husserl and Heidegger, in a dialogue with the respective analysis of J. Derrida. By means of a reading that recognizes continuities and deployments, we will seek to demonstrate that the practical elaborations of the touch developed by Derrida articulate an understanding in certain continuity with those traditional elaborations, in the context of a singular reading of the respective texts.

Keywords: touch; hand; Husserl; Heidegger; Derrida.

Sumario: 1. Introducción; 2. La mano y el tacto en elaboraciones de Husserl; 2. a. El tacto y los movimientos como vías para el conocimiento de sí; 2. b. La cuestión de la empatía; 3 . Heidegger y los gestos manuales; 3. a. La mano y sus significaciones; 3. b. Consideraciones de la inter-corporeidad; 4. El tocar y la corporalidad en recorridos de Derrida; 4. a. Tangente en la mano de Husserl; 4. b. Tangentes en la mano de Heidegger; 4c. El tocar derrideano; 5. Consideraciones finales; 6. Referencias bibliográficas.

Cómo citar: Butierrez, L. F. (2021): "Fronteras táctiles. Perspectivas en torno a la mano y el tacto en elaboraciones de Husserl, Heidegger y Derrida”, en Revista de Filosofia 46 (2), 333-353. 


\section{Introducción}

En el campo de las lecturas e interpretaciones de cuestiones relativas a la corporeidad en el pensamiento filosófico contemporáneo podemos distinguir, entre otras, dos líneas de investigación: aquellas que abordan continuidades y rupturas con la tradición fenomenológica de Husserl y las que despliegan la recepción francesa respectiva, a partir de las consideraciones del cuerpo vivo en relación a la noción de carne (Chair). En este marco, se inscriben algunas consideraciones específicas de la corporalidad: las cuestiones relativas al cuerpo propio, la espacialidad, los sentidos y, en particular, la mano y el tacto. Con frecuencia, podemos encontrar allí abordajes que se articulan en análisis comparativos desde un enfoque progresista y superador respecto de la tradición fenomenológica, en especial, considerando las recepciones de Merleau Ponty, Jean Luc Nancy y Jacques Derrida ${ }^{2}$.

En el siguiente artículo proponemos un recorrido comparativo, en el marco de los desarrollos en torno al cuerpo de Husserl, Heidegger y Derrida. Específicamente, nos detendremos en sus perspectivas de las cuestiones hápticas ligadas a la mano, pues consideramos que allí se ponen de manifiesto y articulan sus propias comprensiones sobre la corporeidad. Por la vía de una lectura que busca apartarse de aquél enfoque progresista-superador de ciertas recepciones contemporáneas, buscaremos demostrar que las elaboraciones del tacto desplegadas por Derrida articulan una comprensión en cierta continuidad con las elaboraciones respectivas de Heidegger, en diálogo con las de su maestro. A nuestro entender, la dinámica transicional de la comprensión metafísica del sujeto, que podemos distinguir en los trabajos de Heidegger, es continuada y desarrollada de un modo singular en las elaboraciones respectivas de Derrida, aunque sin ocultar sus limitaciones : dicha dinámica requiere de un recorrido ineludible por la herencia conceptual y semántica de la metafísica occidental.

Para dar cuenta de esta dinámica y continuidad, abordaremos las obras respectivas que consideramos fundamentales para una vinculación en torno al tacto: por un lado, Ideen II de Husserl y Zollikon Seminare, de Heidegger; por otro, Le toucher, Jean Luc Nancy, de J. Derrida. En tal recorrido, nos proponemos circunscribir una descripción y lectura que permita poner en evidencia enfoques, bases comprensivas, continuidades y diferencias.

En primer lugar, especificaremos los análisis de Husserl de la experiencia de la mano y el tacto, articulado con sus consideraciones de la constitución del mundo objetivo, la corporalidad del otro y la empatía. En segundo lugar, abordaremos en el seminario de Heidegger las figuraciones de la corporeidad relativas a la mano, sus gestos y la inter-corporalidad, en un diálogo con las elaboraciones de su maestro. Finalmente, desplegaremos una lectura de los desarrollos respectivos de Derrida a partir de algunas especificaciones: sus abordajes deconstructivos de aquellos textos de Husserl y Heidegger, aunque articulados al modo de un con-tacto singular con la obra de Nancy, a partir de su enfoque sobre el tocar y la corporalidad.

Por medio de ello, buscaremos poner de manifiesto, en general, el estatuto ineludiblemente transicional y fronterizo de estas elaboraciones contemporáneas

En este trabajo discutimos con lecturas que articulan las elaboraciones de tales recepciones destacando notas progresivas, superadoras o desde un giro comprensivo radical, respecto de las limitaciones de los tratamientos metafísicos tradicionales. Al respecto, véanse Llevadot (2013), pp. 556ss.; Monteiro (2014); Ramírez Cobián (2017), pp. 51ss.; Caneda-Lowry (2018). 
y, en particular, el carácter parcial y singular de las lecturas que Derrida hace de aquellos trabajos. De esta manera, planteamos un recorrido por estos pensamientos filosóficos del tacto y la mano, con lo cual también esperamos destacar sus aportes relevantes para los análisis contemporáneos en torno a la corporalidad.

\section{La mano y el tacto en elaboraciones de Husserl}

En efecto, podemos encontrar consideraciones fundamentales sobre el tacto y la mano en su obra elaborada por varios años: Ideen II. Allí, Husserl desarrolla un análisis de la constelación conciencia-cuerpo-mundo por diversas vías, entre las cuales destacamos el paso del yo puro al yo personal, aunque poniendo de manifiesto su carácter corpóreo y situacional.

La distinción fundamental de la que parte, en sus diferentes análisis de la corporalidad, remite a las dos manifestaciones del cuerpo: es un cuerpo físico como cualquier otro (Körper) y también un cuerpo orgánico (Leib) vivido "desde dentro"3. Específicamente, los desarrollos fundamentales de estas cuestiones pueden situarse a partir del parágrafo 35 de la segunda sección, donde Husserl despliega un análisis de la realidad natural del hombre desde los vínculos entre la vida anímica y corporal, revelando las imbricaciones en el mundo humano compartido. Partiendo del yo puro en su corriente de vivencias, distingue tres estratos: material, anímico y espiritual ${ }^{4}$.

Comenzando su análisis por el cuerpo orgánico, vivo y propio, subraya el carácter vinculante pues, a diferencia del cuerpo físico, el cuerpo propio comparte y articula elementos de aquellos estratos. Esta comprensión tiene dos desarrollos correlativos: por un lado, desde un enfoque natural-material-perceptivo y, por otro, a partir de una perspectiva personalista que atiende las relaciones culturales, axiológicas y comunitarias, de tal modo que permita poner de manifiesto el entramado interrelacional del mundo circundante (Umwelt).

A continuación abordaremos el desarrollo de aquél enfoque material-perceptivo a partir de su análisis de la experiencia de la mano, el tacto y su vinculación con el resto de los sentidos. Luego, presentaremos algunas puntualizaciones que realiza en torno a la corporalidad del otro, en el marco del carácter intersubjetivo que distingue en la constitución de un mundo y naturaleza objetiva, para dar cuenta de aspectos problemáticos en la consideración de la empatía. De esta manera, buscamos evidenciar supuestos desde la comprensión en la que se articulan estas consideraciones, en vistas de una vinculación posterior con el enfoque de Heidegger.

\section{2. a. El tacto y los movimientos como vías para el conocimiento de sí}

En continuidad con las elaboraciones fenomenológicas de las cinestesias (Kinästhese) en sus trabajos anteriores ${ }^{5}$, Husserl desarrolla el análisis de lo que denomina ubiestesias (Empfindniss $)^{6}$, en referencia a las sensaciones que porta el

3 Husserl CM, pp. 127-9[159-61]. Las citas de Husserl y Heidegger indican las abreviaturas de las obras, la paginación del texto original y de la traducción utilizada, cuyas especificaciones se encuentran en el apartado de bibliografía.

$4 \quad$ Véase Husserl Ideen II, pp. 109s. [145ss]; 137-43[175-82].

Véase Husserl DuR, pp. 160ss.

6 Aquí seguimos este neologismo a partir de la traducción de Antonio Zirión. El término utilizado por Husserl es 
cuerpo y se localizan en determinadas zonas del mismo, las cuales se distinguen de las sensaciones de movimiento y de las vinculadas a los sentimientos sensibles ${ }^{7}$. Aquellas sensaciones permiten experienciar el cuerpo desde sí mismo, en una doblez que lo diferencia de la manifestación meramente material.

En este marco, prioriza el tacto y la palpación del cuerpo, por sobre el sentido de la vista y el oído. En especial, presenta el ejemplo de la mano como el que mejor articula estos análisis sobre el cuerpo, a partir de un conjunto de consideraciones: a) la doble aprehensión, b) la inmediatez, c) la localización táctil, d) los aspectos no materiales implicados en el fenómeno del tacto y e) el movimiento espontáneo.

En primer lugar, describe la experiencia del tocar de la mano derecha sobre la izquierda. Allí se revela que las sensaciones táctiles y de movimiento pertenecen tanto a una como a otra, lo cual le permite destacar la vivencia de sí como cuerpo: toco y me experimento corpóreo en el tocar. Esta experiencia se muestra privilegiada pues permite articular las dos dimensiones de la corporalidad, es decir, evidencian el cuerpo vivo que experimenta el mundo, así como también su propio carácter corpóreo-material. Ello remite a la doble aprehensión tocante-tocado que supone, como veremos, cierta inmediatez.

Luego, destaca que la sensación de toque tiene una localización corporal propia. En tales figuraciones de la doble aprehensión, la consideración del tacto remite a localizaciones preferenciales del cuerpo material: la mano y los dedos se presentan como una localización fundamental del tacto (Husserl Ideen II, pp. 145s. [185]). Precisamente por esta vía, destaca que el toque es un suceso físico que se distingue del tocarse de las meras cosas materiales. En tal sentido, como fenómeno de la doble aprehensión, describe las sensaciones que (aún) resuenan cuando el objeto tocado y la mano se separan en el palpar.

Desde aquí podemos distinguir como supuesto la consideración de la inmediatez háptica y su jerarquía correlativa, en especial, cuando Husserl diferencia el objeto externo y el cuerpo, para resaltar la sensación táctil: en el primero, ella se aprehende como nota del objeto y, en el segundo, como sensación del cuerpo. Esto es una diferencia fundamental respecto del sentido de la visión que no puede auto-percibir el ojo, es decir, que no cumplimenta una doble aprehensión posible. Del mismo modo se distingue del oír, donde no hay localización física de la sensación auditiva del sonido. Esta eminencia del sentido del tacto lo conduce incluso a sostener que aquellos sentidos pueden considerarse como un objeto táctil para la mano.

Este realce táctil se halla implícito también en la diferenciación entre las propiedades de las cosas reales (constituidas desde un esquema sensible y matizaciones respectivas) y las ubiestesias táctiles, las cuales no se dan en esquemas sensibles, sino de un modo relativo al flujo de la experiencia. En general, los esquemas se vinculan a un proceso de determinación objetiva en la experiencia perceptual, que media entre la cosa u objeto espacial y la experiencia (subjetiva) de él ${ }^{8}$.

Pero el cuerpo vivido remite a una unidad de experiencia articulado en

sinónimo de Empfindung (sensación). Véase Husserl Ideen II, pp. 144-7[184-7].

A este respecto, encontramos un esclarecedor análisis comparativo en San Martin (2010), pp. 182-5.

8 El esquema sensible es un estrato que se repite en cada aparición de la cosa y que mantiene idéntica su forma frente las múltiples manifestaciones fenomenológicas condicionadas subjetivamente, lo cual remite a una idea de la cosa que guía el proceso de percepción. Al respecto, véase, Husserl Ideen I, pp. 350s. [361s. ]; Husserl, Ideen II, pp. $37 \mathrm{ss}$. [67s. ]; 75[107s. ]. 
circunstancias y al entorno 9 . A diferencia de los cuerpos solo materiales, aquél puede tener sensaciones dobles de auto-aprehensión en un esquema corporal, es decir, un esquema que articula las sensaciones y la aprehensión del propio cuerpo viviente ${ }^{10}$. Esta articulación se expresa en experiencias intuitivas: la mano en la experiencia táctil se liga con la posibilidad del cuerpo sentido y vivido, manifestándose de un modo inmediato como mi cuerpo, esto es, con el carácter de plenitud intuitiva. Ello permite explicar el fenómeno donde puede "desaparecer" lo real material, pero no lo vivido corporal del mismo como, por ejemplo, el caso de una negación material de experiencia corporal, una apariencia (Apparenz), etc. ${ }^{11}$. Estas puntualizaciones subrayan la constitución del cuerpo vivido en la inmediatez de la tactualidad por sobre los demás sentidos ${ }^{12}$.

En general, aquella correlatividad perceptiva en la aprehensión supone el carácter irreductible de la corporalidad en la experiencia, es decir, que no es posible comprender una experiencia sin cuerpo orgánico y físico. En este marco, el estrato primario del cuerpo en el mundo abre una espacialidad y posibilidad de orientación, desde la cual se dan las relaciones con los objetos en el mundo circundante ${ }^{13}$.

Finalmente, en lo que respecta al movimiento espontáneo, analiza las relaciones entre cuerpo y voluntad. En ese marco, destaca que la potencialidad ligada al movimiento también influye en la modalidad de manifestación de los objetos en la experiencia, es decir, no solo imprime variabilidad en las perspectivas, sino que también permite dar cuenta del carácter transformador del cuerpo vivo en el mundo circundante, donde el sujeto puede comprenderse como contramiembro de la naturaleza material (Ibíd., pp. 152[191s]). En tal sentido, Husserl vincula a la expresión yo puedo esta capacidad operatoria del cuerpo ${ }^{14}$, es decir, en continuidad con sus elaboraciones específicas sobre injerencias prácticas y activas en el mundo circundante ${ }^{15}$.

Sin embargo, aún cuando estas elaboraciones de Husserl parecen darle al cuerpo propio un lugar eminente en el conocimiento de sí, no deja de reconocerle limitaciones. Entre ellas, destaca la constitución corporal imperfecta a partir de aquellas partes que acortan o limitan la perspectiva: la materialidad del cuerpo también obstaculiza la perspectiva de sí, cancelando la posibilidad de auto-accesibilidad total (Ibíd, pp. 159 $[199])^{16}$. Precisamente por ello, el acceso al cuerpo propio puede ampliarse a partir de la experiencia con el cuerpo del otro.

$9 \quad$ Véase Ibíd., pp. 40s. [70s. ].

10 Merleau Ponty desarrolla un conjunto de elaboraciones respecto del esquema corporal que son fundamentales para una fenomenología de la corporalidad, no solo en diálogo con estas especificaciones en Husserl, sino también con la analítica heideggeriana en torno a la estructura de ser-en-el-mundo del Dasein. Las respectivas relaciones entre esquema corporal, movimiento y espacio son desarrolladas inicialmente en Merleau Ponty PP, pp. 114-172[115-164].

11 Véase Husserl Ideen II, pp. 115ss. [151ss. ].

12 En este sentido, sostiene: "El cuerpo solo puede constituirse primigeniamente como tal en la tactualidad y todo lo que se localiza en las sensaciones táctiles como calor, frío, dolor y similares" (Husserl Ideen II, pp. 150s [190]). Cf. El análisis de los sentidos en Muñoz-Delgadillo (2018), pp. 18ss.

13 Véase Husserl Ideen II, pp. 158s [197s].

14 Véase Ibíd., pp. 257-75[304-23]. Cf. Merleau Ponty PP, 160ss. [154ss]

15 Cf. Iribarne (2007), pp. 108ss.

16 Este carácter no totalizable e incompleto del cuerpo propio es una de las vías de continuidad con la recepción francesa. Al respecto, véase Ramos-González, 2014, pp. 213ss. 


\section{2. b. La cuestión de la empatía}

Husserl también examina aquí el darse de los otros ajenos en y desde su corporalidad. Los análisis respectivos los despliega en consideración de la empatía (Einfühlung) y la analogía entre los cuerpos, entendidos como los medios pertinentes para el conocimiento y acceso (parcial) a la corporalidad y al ego del otro ${ }^{17}$. En esta obra, tal despliegue se propone evidenciar la naturaleza objetiva en el marco de la manifestación del mundo compartido.

En efecto, indaga en la espacialidad y posicionalidad de los cuerpos para distinguir la protopresencia (en referencia a las vivencias del objeto dado en una presencia primigenia) de la apercepción y empatía: en la experiencia del otro como cuerpo le transfiero por empatía las sensaciones vividas propias, coordinando lo físico y lo psíquico. Por esta vía, se pone de manifiesto una copresencia corporal que no arroja datos acabados: la vida anímica del otro no posee la originariedad del cuerpo propio.

Pero estas afirmaciones ponen de manifiesto algunas tensiones. Por ejemplo, con las ubiestesias, el darse co-originario se manifiesta en el contacto con la mano del otro, estableciendo con ello fronteras algo difusas para la distinción de los cuerpos: puede ponerse de manifiesto aquí una corriente de vivencias originaria e indiferenciada, donde el cuerpo del otro es percibido del mismo modo que el propio. Como sostiene Agamben, el carácter problemático de la empatía, abordado en la época por Theodor Lipps, se pone de manifiesto por medio de la distinción de elementos de alteridad en la propia esfera del ego, lo cual puede tornar problemático el axioma de la originariedad del cuerpo propio, en tanto mio, con su plenitud intuitiva correlativa ${ }^{18}$.

Aun el marco ambiguo de estas distinciones, Husserl destaca que solo en la experiencia objetiva del cuerpo del otro (esto es, si se considera el enlace y empatía de dos lados) puede manifestarse la realidad objetiva corporal y de la naturaleza, a través de la objetividad-intersubjetiva de la cosa experimentada. Más en detalle: bajo la experiencia subjetiva del cuerpo y del mundo subyace un estrato intersubjetivamente compartido, una normalidad o estructura sensitivo-perceptiva que permite la confluencia del sentido relativo (del sujeto de la experiencia) con el objetivo (es decir, en el marco de un estrato relativo a una comunidad intersubjetiva) ${ }^{19}$. Como vemos, estos análisis se enmarcan en una dimensión gnoseológica a partir de la puesta entre paréntesis del mundo, independiente de la vida humana ${ }^{20}$. Ello se vincula, en definitiva, con el horizonte de cientificidad al que se orientan sus indagaciones y las restricciones que ello le impone.

Ahora bien, estas especificaciones en torno a la originariedad del cuerpo propio y la experiencia del mundo objetivo, han suscitado ciertas lecturas e interpretaciones posteriores con un énfasis parcial puesto en alguno de los polos implicados. Entre ellas, pueden encontrarse recepciones que destacan de manera independiente las dinámicas constitutivas e "internas" de la esfera del ego $^{21}$. En particular, el énfasis puesto en el polo constitutivo del ego y la intuición plena permiten subrayar, por contraste, el carácter parcial y mediato del conocimiento del cuerpo ajeno, lo cual no deja de presentar algunas tensiones.

\footnotetext{
17 Asimismo, pueden encontrarse breves elaboraciones sobre la percepción de la mano en el cuerpo ajeno, en las dos primeras partes de Zur Phänomenologie der Intersubjektivität [1905-20 (Hua XIII); 1921-28 (XIV)].

Agamben (2019), pp. 64ss. Cf. Stein (2005), pp. 22ss.

Véase, Husserl Ideen II, pp. 169s. [211ss. ].

Aquí seguimos la lectura de Presas (1983), p. 50. Cf. Depraz (1995), pp. 122-5.

Adrián (2013), pp. 19-27.
} 
En línea con estas lecturas parciales, a partir de las elaboraciones complementarias de Husserl de las síntesis activas y pasivas ${ }^{22}$, Breuer distingue un exceso afectivo que se desprende de la confluencia entre los estímulos de las cosas y la atención afectiva que despiertan ${ }^{23}$. Según su interpretación, tal exceso prefiguraría la experiencia con los objetos en el mundo. A nuestro entender, la parcialidad de este tipo de lecturas reside en que pueden descuidar los aspectos correlativos en las especificaciones respectivas.

El carácter constitutivo de la conciencia, implícito en la modalidad en la que las cosas se donan en la experiencia, co-implica la unidad o identidad inherente a la cosa misma ${ }^{24}$. En tal sentido, otras lecturas ponen énfasis en esta co-implicación, destacando que el mundo se manifiesta como horizonte último al permitir que los objetos mundanos aparezcan con el significado que tienen ${ }^{25}$. Aquí subrayamos que la dinámica de constitución del mundo objetivo supone diversas instancias entrelazadas: subjetividad, mundo de la vida, cuerpo e intersubjetividad. Precisamente en el marco de tal entramado puede entenderse como problemático el supuesto contraste nítido entre el conocimiento de sí (con su plenitud intuitiva) y el del cuerpo ajeno.

En suma, los supuestos en el análisis del tacto manual, así como el enfoque desde el ego y la corporalidad con su plenitud intuitiva respecto del acceso al cuerpo del otro, pueden distinguirse como herencias de una comprensión ligada a la tradición moderna, en especial, por sus aspectos jerárquicos y relativos a la presencia en su inmediatez, los cuales, en definitiva, suponen el carácter eminente y central de la subjetividad, la conciencia o el ego para los análisis. Entre las variadas discusiones que ello ha suscitado, destacamos a continuación ciertos análisis de Heidegger, especialmente en relación a la mano.

\section{Heidegger y los gestos manuales}

En el caso de la obra de Heidegger, encontramos dos tratamientos relevantes sobre el cuerpo: uno breve, en Sein und Zeit (SuZ) y otro más descriptivo, en sus Zollikoner Seminare (ZS). En el primer caso, Heidegger esboza una comprensión de la corporeidad como modo originario de ser en el mundo, desde lo cual reconoce la necesidad de un análisis especifico ${ }^{26}$. En línea con Husserl, rechaza la posibilidad de pensar un existente humano sin su cuerpo, en el marco de una articulación irreductible con el mundo circundante y en su entramado respeccional de significatividad. En la analítica del Dasein destaca que la comprensión y significatividad son co-estructurales con el ser-en-el-mundo, determinando las modalidades de aprehensión de las cosas y de proyección en las relaciones respectivas. En este sentido, los desplazamientos

22 Aquí, el marco general es el proceso de formación de sentido. En la génesis activa el sujeto es productivo en la constitución de los objetos; en la segunda, se pone énfasis en la pasividad por la que uno se ve afectado de antemano, involuntariamente, por hábitos, patrones motores, disposiciones, emociones, recuerdos, etc. Véase, por ejemplo, Husserl, $A S$, pp. 3s. Cf. Husserl, AzpS.

23 Sobre el esquema corporal y su rol en el conocimiento de sí y en la constitución del mundo objetual-espacial, véase Breuer (2015), pp. 120-7.

24 Aquí seguimos lecturas recientes de Husserl que subrayan una transición de los enfoques tradicionales, por medio de la asignación de validez/sentido en una co-relación de sujeto y mundo, en Villanueva-Barreto (2012), pp. 14s. Cf. Husserl, $A S$, pp. 20-6.

25 Al respecto, véase Zahavi (2004), pp. 59s. Cf. Bernet (2004), pp. 160s.

26 Véase Heidegger SuZ, pp. 107s. [133s. ]. 
en dichas estructuras son correlativos con las modificaciones en la manifestación remisional y el ocuparse en el trato circunspecto. De esta manera, en SuZ el tema del cuerpo no es abordado de un modo directo ni de modo insuficiente, lo cual ha suscitado diversos estudios críticos posteriores ${ }^{27}$.

En el segundo trabajo, podemos encontrar un tratamiento más detallado a partir de la distinción de la corporalidad viva (Leiblichkeit), cuyos principales antecedentes se encuentran en las elaboraciones de su maestro. En este marco, sus consideraciones del campo háptico se circunscriben principalmente a un análisis del gesto.

En el marco del conjunto de su obra, estos seminarios no representan una elaboración directa de Heidegger, más bien se desarrollan en un contexto de diálogo y discusión con un grupo de especialistas en el ámbito médico. En efecto, tales encuentros se originan en un intercambio epistolar con Medard Boss desde 1947, donde ambos se propusieron especificar y comprender las cuestiones inherentes al Daseisnanalyse en la tradición de Ludwig Binswanger. En ese marco, Boss lo invito a unas conversaciones con un conjunto de colegas y estudiantes de psiquiatría y psicología en su casa en Zollikon, desde 1959. Lo que Heidegger promovió en tales encuentros es la experiencia de un pensar, ver y decir otro, respecto a las cuestiones consideradas, a partir de los interrogantes de los asistentes en torno a la ausencia de la temática del cuerpo en su obra. Desde allí, el diálogo se orientó a la posibilidad de integración de la ontología heideggeriana a la práctica clínica, lo cual permitió una tematización directa de la Leiblichkeit no presente en SuZ.

En efecto, allí Heidegger da cuenta de un sentido originario de la corporeidad que no se agote en el cuerpo mensurable y objetivable (Körper), reducido a un mero estar presente (vorhanden). De este modo se propone dar cuenta del Dasein en su apertura al mundo y sus correlaciones, más allá de la reducción a su aspecto material en la perspectiva científica tradicional.

A continuación desarrollaremos las especificaciones de fenómenos corporales que nuestro autor propone para dar cuenta de tal estatuto, haciendo especial hincapié en las figuraciones de la corporeidad relativas a la mano y sus gestos. Luego, abordaremos brevemente sus consideraciones de los vínculos con el cuerpo ajeno, en el marco de aquello que distinguimos como una posición transicional respecto al enfoque de Husser ${ }^{28}$.

\section{3. a. La mano y sus significaciones}

En efecto, en estos seminarios, Heidegger dialoga y establece distinciones respecto a los sentidos: el ver y oír dirigidos al mundo; al agarre o el tocar; el quedar absorto en una cosa, donde el cuerpo se manifiesta como fenómeno de privación, etc. En este conjunto de manifestaciones corporales se propone poner en evidencia sus respectivos entramados inter-relacionales y de significación.

En continuidad con Husserl, destaca la doble aprehensión de la mano como diferente respecto del sentido auditivo o visual: se puede percibir la mano desde "adentro", al tiempo que se establece un contacto directo con lo tomado. Frente a ello, el ojo no está en contacto directo con lo visto ${ }^{29}$. Pero con ello subraya que el

\footnotetext{
Véase De Waelhans (1963), pp. xviii-xix.

Véase Butierrez (2020), pp. 89ss.

Ibíd, pp. 107ss. [141ss. ].
} 
sentido de proximidad (Nah-Sinn) relativo al tacto, se opone al sentido de la lejanía (Fern-Sinn) en el ver, tal y como ocurre con la aprehensión corporal en general: en la experiencia, el cuerpo es lo más lejano en la experiencia del espacio.

No obstante, se diferencia de los análisis de su maestro al detenerse en el marco de significación ligado a la mano y el movimiento, en especial, a partir de su consideración del gesto. En efecto, por la vía de una posición crítica de la concepción tradicional del gesto (Gebärde) en el sentido de expresión (Ausdruck) (perteneciente a la representación del cuerpo de la tradición griega), subraya que un movimiento de la mano, como saludar, pertenece al ser de la mano y no a una mera expresión de interioridad, estado mental o afectivo. En tal sentido, el movimiento mismo de la mano ya es el significado del saludar. De esta manera, el gesto revela un movimiento del ser-en-el-mundo, formando parte inherente al espacio y la corporeidad del Dasein ${ }^{30}$. Así, el gesto remite al comportarse (Betragen) del hombre como ser corporal en el mundo, en su relación irreductible con los otros entes ${ }^{31}$.

Aquella imbricación, por ejemplo, figura en la expresión somos todo oídos en una conversación: corporalmente participamos en el diálogo, co-ejecutando el diálogo por el oír-hablar con el que participo, por ejemplo, al estar sentado en la silla para poder ser todo oídos ${ }^{32}$. De esta manera, subraya el sentido extático de la corporalidad, el cual parece no requerir de una remisión exclusiva al tacto. Entendido aquí como una tentativa transicional de la perspectiva cientificista de su maestro, Heidegger busca poner en evidencia las relaciones de significación en la comprensión, donde cuerpo y espacio se entretejen: el significado de un gesto remite a modalidades del ser-en-el-mundo. Tales remisiones también se manifiestan en las relaciones entre los cuerpos.

\section{3. b. Consideraciones de la inter-corporeidad}

En el marco de la modalización que imprime la comprensión, nuestro autor destaca que el corporar (Leiben) (modalidad verbal del estar-siendo-cuerpo) se determina a partir del modo del ser del Dasein ${ }^{33}$. En este marco, nuestra interpretación toma distancia de aquellas lecturas que enfatizan la anterioridad ontológica de la apertura del ser en el corporar, respecto de la correlatividad constitutiva histórico-social del Dasein en su corporeidad ${ }^{34}$. A nuestro entender, el corporar, como modalidad de manifestación del cuerpo en tanto es cuerpo, pertenece al ser-en-el-mundo co-determinado en el estar-abierto y el tener mundo, es decir, se manifiesta interdependiente de las relaciones con y en el mundo, en su ligazón con la experiencia en el tiempo. Por ello, distinguimos que estas elaboraciones tampoco remiten a una modalidad general de todo cuerpo en su esencia, sino a la modalidad de ser cuerpo del Dasein ${ }^{35}$, en vistas de la delimitación del modo apropiado para interrogar el fenómeno del cuerpo.

En este marco, el límite del corporar es el horizonte del ser en el cual permanecemos $^{36}$. En este marco relacional, todo el comportamiento del ser humano

\footnotetext{
Véase Ibíd., pp. 116-9[150-3].

${ }^{3}$ Ello pone estas especificaciones en discusión con el tratamiento de la corporalidad en los trabajos fundamentales de Merleau Ponty en PP. Para un análisis comparativo véase Aho (2005), pp. 1-23.

Ibíd, pp. 125s. [160].

Véase Ibíd, p. 113 [147].

Véase Garrido Periñán (2016), pp. 64; 68-72.

Cf. Johnson (2016), p. 139.

Véase Butierrez (2021), pp.230-239.
} 
está determinado por el corporar del cuerpo (incluso todo movimiento del cuerpo en cuanto gesto), no solo en una región ya abierta por la cosa con la que nos relacionamos, sino también en una comprensión del ser determinada. Entre las coestructuras que nuestro autor distingue para tal modalización, destacamos aquí sus consideraciones del ser-con (Mit-sein).

Específicamente, en la sesión de julio de 1965, analiza la cuestión de la relación inter-corporal, tomando distancia de las elaboraciones respectivas de Husserl ${ }^{37}$. En efecto, sostiene que hablar de relación interhumana conduce al pensar hacia una representación dualista de dos sujetos que están-ahí. Junto a ello, el concepto de relación obstaculiza aprehender el auténtico vínculo que se manifiesta entre los otros. Así, reconoce un constructo implícito en la teoría husserliana de la empatía, en el cual un yo dado primariamente para sí empatiza luego en el otro, descubriéndolo como alter ego.

Frente a ello, Heidegger destaca la estructura del ser-con, entendida como existir con otros en un mutuo referirse a las cosas en la experiencia ${ }^{38}$. Es decir, el serunos-con-otros-en-el-mundo designa la estructura interrelacional del Dasein, que implica el encontrarse con otros en un mismo ser-ahí. Ello no remite a una relación externa entre sujetos, de uno a otro, pues la mera consideración del encuentro entre cuerpos físicos oculta el carácter originario de esta estructura ontológica. En este sentido, entiende que la transferencia supuesta en la empatía y las mutuas relaciones ya presuponen el ser-con y la comprensión del otro como ser humano.

No obstante, desde esta crítica a la perspectiva inicial de Husserl, nuestro autor destaca el posterior abandono de su perspectiva cartesiana, por medio de la referencia originaria al mundo de la vida (Lebenswelt), el cual presenta mayores desarrollos en los trabajos de madurez de su maestro ${ }^{39}$. Encontramos aquí un posible punto de articulación comprensiva, en una dinámica transicional respecto al enfoque de análisis ${ }^{40}$.

De este modo, la distinción originaria de la comprensión y la significación permite destacar las prioridades en lo que respecta a los análisis de la corporeidad en la perspectiva de Heidegger: entre otras implicancias, distinguimos el carácter secundario de los fenómenos corporales del Dasein, en el marco de la distinción de tales dinámicas comprensivas originarias. La figura del gesto permite dar cuenta de una comprensión de la corporeidad con un énfasis puesto en un marco interrelacional, más allá de las reducciones a la mera presencia. Una radicalización de esta comprensión y enfoque podemos encontrarlas en los análisis del tocar que Derrida despliega en torno a la obra de Jean Luc Nancy.

\section{El tocar y la corporalidad en recorridos de Derrida}

Derrida elabora y desarrolla consideraciones sobre el tacto y su historia filosófica a partir de una singular modalidad de tocar la obra de Nancy, con la palabra y la escritura. Aun con una gran influencia del pensamiento de Husserl y Heidegger en su

37 Heidegger ZS, pp. 142-6[177-82].

38 Al respecto, véase un análisis de continuidad con Husserl en Candiotto (2016), pp. 319ss.

39 Véanse los comentarios sobre las elaboraciones de Husserl en Heidegger ZS., pp. 187[224]; 207[246]. Cfr Husserl $D L$, pp. 60-5; 175-8; 200-5, etc.

40 Cf. Inverso (2014), pp. 89-100. 
obra, las cuestiones relativas a la fenomenología de la corporalidad y la corporeidad no ocupan un lugar central en sus trabajos. No obstante, al igual que los ZS de Heidegger, se trata aquí de una elaboración indirecta y dialógica en torno a estas temáticas, en el marco de un singular homenaje a los aportes de Nancy, sin un énfasis explícito sus propias tesis respectivas.

Su punto de partida es la tradición comprensiva que se remonta a Aristóteles, donde entiende que lo háptico no ha conseguido ganar un concepto claro. Precisamente ello le permite desplegar una deconstrucción de esta tradición.

Específicamente, destaca que los desarrollos de Aristóteles dieron comienzo a una tentativa fallida por darle unidad de sentido al tacto y lo tangible, a partir de atención puesta en un supuesto órgano propio de la facultad táctil y su relevancia para la preservación de la vida ${ }^{41}$, perspectiva que domina nuestro pensamiento occidental respectivo ${ }^{42}$.

Desde allí, recorre cierta tradición francesa del tocar donde ubica preferencialmente la síncopa del tocar, como transición comprensiva de las referencias hápticas, donde el contacto se da primariamente en y desde una interrupción. En los comienzos de esta tradición, la eminencia del tacto se manifiesta fundamental, en el marco de una ligazón primaria al conocimiento objetivo: es el único sentido que aporta mayor certeza en la percepción exterior inmediata. Para nuestro autor, esta comprensión inaugural delimita los comienzos del privilegio absoluto en una tradición que entiende como haptocéntrica ${ }^{43}$.

No obstante, distingue un pensamiento posdeconstructivo del tacto, a partir de una comprensión que se aparta de la inmediatez, supuesto inaugural de la fenomenología del tacto. Para ello, Derrida despliega una serie de tangentes o figuras del tocar sobre la tradición respectiva, las cuales no interfieren la superficie del pensamiento en la cual se inscriben, sino que ponen de manifiesto convergencias, disidencias, rodeos y desvíos ${ }^{44}$.

En lo que sigue desarrollaremos tres de estas tangentes que Derrida propone en su obra Le Toucher, Jean Luc Nancy (LT). En primer lugar, analizaremos sus lecturas de las elaboraciones respectivas de Husserl y su inscripción en la comprensión metafísica, junto a ciertas ambigüedades que le permiten plantear la apertura hacia una transición. En segundo lugar, realizaremos un breve abordaje de los aspectos jerárquicos, centralistas y metafísicos que reconoce en las consideraciones (parciales) sobre cuestiones hápticas en textos de Heidegger. Finalmente, puntualizaremos el contacto que propone con la obra de Nancy, el cual entendemos como un singular desarrollo práctico e implícito de su perspectiva sobre el tocar, la mano y la corporalidad.

\section{4. a. Tangente en la mano de Husserl}

Derrida analiza lo que señala como el momento fenomenológico de la historia del tocar, tomando como punto de partida el ejemplo de la mano en Ideen II,

41 Derrida también destaca algunos antecedentes respectivos en el Fedón de Platón. Véase Derrida (2011), pp. 179 ss.

42 Véase, Aristóteles, De Ánima, II, pp. 422b20-424b15.

43 Derrida (2011), p. 72.

44 Los análisis que realiza Derrida sobre la figuralidad del tocar y los suplementos figurales permite ampliar el campo de relaciones del tocar, más allá de su ligazón antropocéntrica con la mano y los dedos. Aquí, la figura de la tangente es una muestra de ello. Véase Ibíd., p. 196. 
donde destaca consideraciones sobre: a) el "yo quiero" y la esfera egológica en su centralidad, b) la inmediatez y c) el carácter espontaneo del movimiento, entendidos como los principales tratamientos de lo háptico en el marco de su análisis de la corporalidad. En ese contexto, se propone poner en evidencia ciertos centralismos, jerarquizaciones y ambigüedades de su escrito, de tal modo que pueda continuarse una vía para la deconstrucción del pensamiento tradicional del tacto.

En primer lugar, destaca la primacía de la mano del hombre por sobre la mano animal en estos tratamientos de Husser ${ }^{45}$. Tal haptocentrismo se apoya en el supuesto de la continuidad espacial y el privilegio de la plenitud intuitiva, es decir, en la relación inmediata y directa entre tocante-tocado, a partir de la mera consideración de la presencia en general. En este sentido, distingue la centralidad dada a la mano y a los dedos en las ejemplificaciones y descripciones de manipulaciones digitales.

En efecto, subraya que el punto de partida de Husserl es la objetividad del cuerpo material, a partir de la epojé egológica como manifestación subjetiva de tal objetividad. Allí, la consideración de los dedos y el tocar, deja fuera su potencial deíctico o de significación. Ello se debe a la relevancia de la simetría de la doble aprehensión para los análisis de Husserl, lo cual permite resaltar el carácter de plenitud intuitiva del fenómeno respectivo.

Si bien Husserl no restringe sus análisis fenomenológicos del ego puro al humano ${ }^{46}$, nuestro autor reconoce que progresivamente el hombre va ocupando mayor lugar en sus análisis, al tiempo que el animal es nombrado marginalmente o entre paréntesis. Esta centralidad puede ser entendida en razón de una jerarquía teleológica o de escala axiológica que se halla en la raíz misma de la fenomenología (Ibíd., p. 244). Sin embargo, destaca que tal privilegio antropocéntrico no es consecuente con la rigurosidad de una reducción trascendental que debe suspender todo juicio de la existencia del mundo.

En segundo lugar, despliega la estructura objetal de la aprehensión, ligada al imperativo de la mano, donde la cosa es determinada en tanto objeto exterior y el objeto interno del tacto. En tal sentido, la inmediatez que se juega en el tocar es espontanea, directa, intuitiva, lo que permite otorgarle una primacía respecto a la visión. Más aún, la relación reflexiva del tacto se logra sin empatía ni apresentación, ni elementos de alteridad: en el tocar, siento inmediatamente desde dentro. Tal plenitud intuitiva se basa en una coincidencia temporal y simultánea de la intuición inmediata en la experiencia tocante-tocado.

En este marco, nuestro pensador francés destaca la exclusión arbitraria en estos análisis de las metáforas usuales que ligan la visión con el tacto. Ello se vincula a la mantención de la primacía del tacto, lo cual Husserl liga con una confusión analógica y en un uso del lenguaje carente de rigor. Estas determinaciones del tocar respecto al sentido común, parecen poner en primer plano la intuición universalizable de las cosas mismas por sobre la experiencia lingüística o discursiva de las mismas. Sin embargo, Derrida entiende que la exclusión de significaciones sedimentadas en el lenguaje, responden más bien a un centralismo trascendental en el discurso de Husserl y no a una consecuencia del rigor metódico de la fenomenología. En este punto especifica como ambigüedad o inconsistencia:

45 Ibíd, p. 235.

46 Tal y como lo específica en sus consideraciones de lo real animal y de la cosa animal en Husserl Ideen II: 177s. [222s. ] 
...en el mismo momento de este repliegue hacia una zona pre lingüística, efectuado en nombre de la reducción y de la intuición, le es preciso exponer el resultado de sus descripciones, justificar y argumentar sus "distinciones esenciales", interpretar sus intuiciones, en verdad, dejarse guiar implícitamente por recortes, distinciones, reparticiones irreductiblemente marcados por la cultura y por el lenguaje . Vemos aquí abrirse el abismo de las cuestiones ligadas al "lenguaje" trascendental y al discurso mismo de la fenomenología (Ibíd., p. 248, el subrayado es del autor).

Tenemos aquí un pasaje fundamental en la deconstrucción derrideana de la fenomenología del tacto: la distinción de un momento del análisis donde se cuela subrepticiamente un significante trascendental ${ }^{47}$, una jerarquía por fuera del marco discursivo que se pretende inamovible.

Luego, realiza una distinción similar en torno a las consideraciones topológicas en los análisis de la localización de las impresiones sensibles y la extensión de las determinaciones materiales de la cosa. En efecto, la indagación fenomenológica respectiva parte del supuesto de una superficie y una interioridad fenomenológica de la mano, radicalmente heterogéneas respecto al espacio y las cualidades reales de la cosa tocada (incluso de las cualidades reales de la mano) (Ibíd, p. 252). Con ello, pone en evidencia otra figura comprensiva que desemboca en el privilegio de lo táctil: el supuesto de una exterioridad para la aprehensión táctil, es decir, un afuera (con su cualidad real de cosa) considerado ajeno a lo tocante y a lo tocado de la impresión fenomenológica. A partir de este espaciamiento heterogéneo, Husserl puede hablar de doble aprehensión en su inmediatez.

Finalmente, analiza la cuestión de la empatía en el marco de su deconstrucción del supuesto de inmediatez. En los análisis respectivos distingue una frontera difusa desde el supuesto de una alteridad inmanente al ego puro. El problema reside en que si en el umbral de lo tocante-tocado hay empatía, y por tanto apresentación analógica, entonces esta doble aprehensión ya no puede ser accesible a una intuición originaria, inmediata y plena, como tampoco lo es el alter ego (Ibíd., p. 257). Desde aquí pone en duda la co-inmediatez intuitiva, a partir de lo cual aborda la necesidad ineludible de suplementos para el cuerpo, tal como especifica:

\begin{abstract}
¿No es necesario que desde el comienzo, en la así supuesta "interioridad psíquica del acto", la visibilidad, la exposición hacia afuera, el rodeo apresentativo, la intrusión del otro, etc. este ya obrando y co-condicione al menos aquello de lo que parece depender y a lo que parece seguir?(...) ¿No es necesario que el intruso este ya instalado? ¿ No es necesario que el espaciamiento dé así cabida al reemplazo? ¿ y que dé cabida al sustituto, al suplemento metonímico, a la técnica? (Ibid, p. 261)
\end{abstract}

Aquí subraya una auto-hetero-afección ligada al espaciamiento y a la espacialidad visible, que se opone a la comprensión de un auto-afección pura e inmediata. Esto le permite pensar una haptología general sin privilegios jerárquicos y la constitución de un cuerpo propio que, en continuidad con la comprensión de Heidegger, supone un paso por lo otro (distinto) de sí.

Encontramos aquí la defensa derrideana del abismo infranqueable entre el yo y el otro como condición irreductible de alteridad. Desde esta perspectiva, el carácter

47 Respecto a este concepto, véase Derrida (1977), pp. 53-67. 
inaccesible que se abre en el encuentro entre mi mano y la mano del otro, por el acceso restringido al cuerpo vivido del otro por apresentación y analogía, constituye un abismo infranqueable que puede pensarse como condición de todo apretón de manos, golpe, caricia y, en general, de la experiencia del cuerpo del otro como tal. En este sentido, subraya una diferencia fundamental con la tentativa de Merleau Ponty por achicar la distancia con la alteridad del otro desde una contemporaneidad original. Ello acarrea el riesgo de reapropiar esta alteridad de un modo violento, por lo cual insiste en retomar la prudencia de Husserl donde el otro no es accesible a una intuición dadora originaria (Ibíd, p. 276). No obstante, esta reserva deja en evidencia ciertas limitaciones comprensivas en relación al acceso al otro: la defensa de la alteridad radical se encuentra aquí con dificultades para dar cuenta del acercamiento al otro, la empatía y otras experiencias corporales, tornando problemática una perspectiva en torno a la inter-corporalidad.

Ahora bien, a partir de aquellas especificaciones, Derrida interpreta la epojé, en su especificación del sentido intencional, como una interrupción ineludiblemente ligada al contacto. Con ello propone una transición comprensiva tras el abandono del supuesto de inmediatez háptica: la epojé abre un espaciamiento y una diferencia interna a lo háptico que es condición para el contacto como tal, aunque por la vía de un rodeo sin posible plenitud inmediata (Ibíd., p. 325). Entendemos que esto se articula en una lectura propia, en el marco de su diálogo con las consideraciones respectivas de Nancy, como (otra) figura de su labor deconstructiva del discurso metafísico $^{48}$.

\section{4. b. Tangentes en la mano de Heidegger}

Del mismo modo, nuestro autor distingue en algunos análisis de Heidegger el privilegio de la mano para el campo háptico, en articulación con el intuicionismo continuista tradicional y un antropocentrismo teleológico, especialmente relativo a la especie animal. A diferencia de las elaboraciones de Husserl, entiende que este privilegio no va en correspondencia con el acto de conocer, sino en relación al entramado de mundo que el gesto de la mano pone de manifiesto. Así, la ejemplaridad de la mano del hombre para dar cuenta del entramado del ser-en-el-mundo, la convierte como la más relevante de las figuras onto-teleológicas y metafísicas (Ibíd., p. 225). Aun así, destaca el esfuerzo por pensar el Leib más allá del cuerpo físico y el organismo vivo $^{49}$. Específicamente, en un trabajo de 1985 y en $L T$, indaga en: a) sus análisis del movimiento gestual de la mano, b)las relaciones con la técnica y c) su distinción del animal como pobre de mundo ${ }^{50}$.

En primer lugar, Derrida subraya continuidades respecto al privilegio de la mano cuyos antecedentes pueden situarse en las elaboraciones en torno a la estructura del ser-en-el-mundo en SuZ, en especial, a partir de sus especificaciones de la interpretación del ente como Zuhandenheit y Vorhandenheit, ambas implicadas en sus análisis de la herramienta (Zeug). Aunque en tales análisis no menciona explícitamente la mano, hace referencia en cambio a términos compuestos que la

\footnotetext{
48 Aquí tomamos distancia de lecturas que entienden este rodeo, en sí mismo, como una de las bases fundamentales del pensamiento de Derrida, en Llevadot (2013), p. 558.

49 Véase Derrida (2011), p. 332, n. 20.

50 Heidegger (1929-30), pp. 274-93[235-50].
} 
incluyen, como manipulación, manejo o maniobra (Hantieren, Hantierung), en el marco de los efectos de la interpretación del ente y las consideraciones sobre la objetivación científica ${ }^{51}$.

Poniendo de relieve las reflexiones heideggerianas en torno al lugar eminente que ha tenido el campo óptico y sus metáforas en la tradición metafísica, nuestro pensador francés insiste en el carácter accesorio de estas tentativas jerárquicas de los sentidos ${ }^{52}$.

En segundo lugar, en un breve trabajo de 1985, ofrece un ensayo específico sobre la mano de/en Heidegger. Allí la mano se torna definitoria de lo que es el hombre, respecto del ser de otras especies, pues se relaciona estrechamente con el pensamiento, la técnica y la adquisición de conocimientos. Nuestro autor parte de la observación de un conjunto de fotografías de época donde Heidegger posa escribiendo con una pluma. En esta modalidad artesanal de presentación de su imagen, no solo afirma el carácter eminente de la mano, sino también la consideración secundaria de la escritura. En este marco, cita un análisis de Heidegger donde compara la mano humana con la del mono, donde destaca que éste posee órganos de prensión pero no posee mano, es decir, carece de las estructuras entramadas del ser-en-el-mundo ${ }^{53}$. En tal distinción, Derrida reencuentra la inscripción reductora de la animalidad en el círculo cerrado de sus programas biológicos, sin atender el amplio bagaje del saber zoológico respectivo, lo cual se enmarca en sus análisis de finales de la década de 1920 en torno a la pobreza de mundo animal.

En suma, este pensador francés entiende que la consideración heideggeriana de la mano reúne los caracteres del logocentrismo, fonocentrismo y haptocentrismo que subyacen a las elaboraciones respectivas de Husser ${ }^{54}$. A nuestro entender, ello puede leerse como una apropiación afín a los desarrollos de su propia perspectiva, más que a las dinámicas internas de las elaboraciones de Heidegger ${ }^{55}$. En tal sentido, nuestro autor retoma desde aquí la perspectiva transicional que reconoce en la obra de Nancy.

\section{4c. El tocar derrideano}

En un dinámico juego de palabras, Derrida se propone desarrollar en $L T$ un tocar figurativo del trabajo de Nancy, por la vía de un comentario y análisis de sus elaboraciones sobre el tacto. En este marco, la perspectiva de nuestro autor remite a un contacto de escritura a escritura, como un tocar tangencial que colabora con el propio movimiento de Nancy, en su multiplicación y apertura respectiva. Esta figuración del tocar derrideano se halla implicado en un gesto propio de amistad, saludo y homenaje a su par francés. Esta modalidad de contacto, más que a la perspectiva política de Derrida, remite al horizonte de su comprensión de la alteridad, tal como figura en las tangentes antes analizadas ${ }^{56}$.

51 Cf. Heidegger SuZ, pp. 67-76 [94-103]. Aquí reencontramos la falta de tematización de la corporalidad: en estos parágrafos de $\mathrm{SuZ}$, las prácticas y hábitos corporificados exigen la presencia de un cuerpo. Pero el Dasein aquí no tiene cuerpo y el cuerpo vivido, necesario para tales actividades, no es tematizado.

52 Para un análisis de la metáfora fotológica en la tradición metafísica, véase Derrida (2012a), pp. 42-4.

53 Derrida (1985), p. 510. Cf. Heidegger (1951-2), p. 18.

$54 \quad$ Derrida (1985), p. 519.

55 Cf. Zizek (2018), pp. 72ss.

56 Cf. Monteiro (2014), pp. 109-12. 
En este marco, los análisis se despliegan respecto de: a) las consideraciones sobre el supuesto de inmediatez y la síncopa constitutiva de la doble aprehensión; b) la tentativa de deconstrucción del cristianismo implicado en la traducción francesa de Leib por Chair (carne) y c) el supuesto tradicional de la unicidad de sentido táctil. Tales cuestiones permiten desplegar un enfoque de lo mesiánico ligado al pensamiento del tocar como don, al tiempo que establecen el marco de una comprensión de la corporalidad correlativa a una perspectiva sobre la ecotecnia, la téchne y los suplementos proteicos para el cuerpo.

Por un lado, Derrida sigue el rastro de las elaboraciones sobre la síncopa y la no-continuidad constitutivas del tocar, distinguiendo especialmente la consideración nanciana del límite de lo tocado como un espaciamiento infranqueable, inaprehensible e inapropiable ${ }^{57}$. Asimismo, por medio del uso que Nancy hace del supuesto tradicional de partes extra partes $^{58}$, logra extremar su rechazo de la inmediatez, en una apertura del tocar a la divisibilidad diseminal. Desde allí, la ruptura con la contigüidad del contacto permite pensar el intervalo del espaciamiento de un modo inaprensible e irreductible (Ibíd., p. 179). Por esta vía deconstructiva de la rigurosidad de todo concepto de lo háptico, se propone continuar el pensamiento nanciano del tocar en su aspecto local, modal y fractal ${ }^{59}$.

En lo que respecta al privilegio tradicional del tocar y de la mano, destaca la interpretación de Nancy donde la preeminencia se articula más bien con una comprensión del tacto caracterizado por no subordinar nada a él mismo, esbozando así una tentativa de des-jerarquización que lo separe de la tradición haptofundamentalista de Kant y Husserl (Ibíd., p. 103). En este sentido, entiende que todos los sentidos están incluidos en el corpus del tacto, como el mirar, escuchar, olfatear, saborear, etc.

Por esta vía, Derrida distingue la necesidad de una distancia de la herencia metafísica articulada en el vocabulario tradicional del tacto, pues los compromisos no tematizados con ella obstaculizan el despliegue de dicha comprensión. A diferencia de ciertas lecturas respectivas, entendemos que tales distinciones le permiten ponerse en guardia contra la tradición apropiante del tacto, al tiempo que reconoce/preserva la distancia irreductible en el con-tacto de la escritura ${ }^{60}$. En tal sentido, insiste en la diferencia irreductible entre lo tocante y lo tocado, tal y como se pone de manifiesto en la caricia que toca con lo mesiánico, es decir, con aquello por-venir que quiebra su ligazón con lo dado a la presencia y al campo cerrado de la representación ${ }^{61}$. Con ello, también enfatiza el carácter inapropiable del tocar y abierto al don, es decir, a la proximidad que simultáneamente da (sin obtener nada) y retira su ser-presente en el $\mathrm{dar}^{62}$.

57 De lo cual, especifica : "El límite no se toca, no se deja tocar, se sustrae al tocamiento, que o bien no lo alcanza nunca, o bien lo transgrede para siempre", Derrida( 2011), p. 25.

58 Véase, Nancy (2010), p. 24s. Como uno de los supuestos fundamentales de la inmediatez, tal supuesto remite a la concepción de la materialidad en la que cada parte componente es externa a la otra. Lo contrario sería la autopenetración de la sustancia o de la materia entre sí.

59 Al respecto, véase Derrida (2011), p. 228. Cf. Nancy (2010), p. 68.

60 Cf. Caneda-Lowry (2018), pp. 33ss.

61 Su perspectiva general de la mesianicidad remite a una estructura de la experiencia (no exclusivamente religiosa) en la cual el carácter por-venir del acontecimiento se liga con una alteridad radicalmente heterogénea, respecto al campo cerrado de la representación o el sujeto, es decir, de un modo no previsible ni calculable. Al respecto, véase Derrida (2012b), pp. 42; 73; 89.

62 Véase, respectivamente, Derrida (2011), pp. 121; 245. 
Por otro lado, la consideración de la carne (chair) como una tentativa francesa de traducción del Leib husserliano, llevan a Nancy a una deconstrucción del cristianismo que nuestro autor sigue de cerca en su obra, pues entiende que la comprensión respectiva se mantiene en el pensamiento occidental ${ }^{63}$. En especial, a partir de la obra Corpus, sigue su análisis de la carne de Cristo y el tocar espiritual que atraviesa la narrativa bíblica, donde el cuerpo físico ocupa un lugar menor respecto a un enfoque trascendental y espiritual de la corporalidad, pensamiento que entiende deudor de un antropo-teleologismo y centralismo de la mano del hombre (Ibíd., p. 344).

Finalmente, en sus elaboraciones y lecturas posheideggerianas sobre la ecotecnia y la téchne de los cuerpos ${ }^{64}$, nuestro autor destaca un pensamiento emergente de la experiencia de trasplante de corazón de Nancy, la cual no solo lo ha convertido en un superviviente (su sobrevida pos-trasplante ha superado con creces la estipulada por sus médicos), sino que también ha implicado una singular experiencia de alteridad: Nancy no sabe si el nuevo corazón pertenece a un hombre o una mujer ${ }^{65}$. Es precisamente desde aquí donde subraya la téchne de los cuerpos en su carácter suplementario, a partir de aquella consideración del desplazamiento, apartamiento y espaciamiento irreductible que disloca la tradición continuista: el cuerpo que se busca y que busca tocarse es originariamente y esencialmente receptivo de injertos, prótesis, sustituciones, etc.

Por medio de estos con-tactos, Derrida reconoce el carácter ambiguo de la relación de Nancy con la tradición metafísica haptocéntrica pues, si en parte desarrolla una vía de transición, no considera que aquella pueda superarse de un modo acabado o radical ${ }^{66}$. En este marco, destaca su elaboración no intuicionista, ni continuista del tacto, a partir de la discontinuidad, la interrupción y la síncopa en su respectiva comprensión, de modo tal que, sin unificarse en un sentido propio, el tocar puede pensarse como diseminal y múltiple.

En suma, estas lecturas en diálogo con la obra de Nancy le permiten a Derrida, no solo circunscribir una tradición comprensiva de la corporeidad a partir de aquellas figuras tradicionales del tocar, sino también aludir implícitamente a su propia comprensión en torno a las cuestiones hápticas, desde la deconstrucción que allí despliega. En este sentido, entendemos tal procedimiento deconstructivo como una figuración práctica de su comprensión del tacto, el tocar y la corporeidad a partir de una relación inter-textual. Junto a ello, interpretamos que estas modalidades de abordaje, con sus énfasis y distinciones, dan cuenta de una apropiación de aquellas elaboraciones en vistas de lograr un desplazamiento de la respectiva tradición metafísica y su comprensión de base, sin una consideración exhaustiva de las dinámicas y desplazamientos en las obras de Husserl y Heidegger.

No obstante, tal figuración derrideana desarrolla un vía posdeconstructiva del pensamiento tradicional del tacto, que si bien insiste en la tentativa de des-centralizar el supuesto de la inmediatez y la primacía de la presencia, luego de subrayar el riesgo de apropiar la alteridad del otro en tanto tal, parece no dar cuenta de un modo suficiente de las cuestiones en torno a la inter-corporalidad.

\footnotetext{
63 Considerando la recepción de Merleau Ponty, Derrida reconoce un punto intraductible de la diferencia entre Leib y Körper, que el término Chair no puede subsumir. En este marco, distingue una historia de la corporalidad en la que también se juega una tradición europea que discute con el cristianismo, en Derrida (2011), p. 201.

64 Para la interpretación de la ecotecnia como nombre ontológico de la técnica en Heidegger, véase Bulo-Vargas (2012), pp. 60s.

65 Ibíd., pp. 147ss. Cf. Nancy (2006), pp. 14-9; 31.

66 Véase Derrida (2011), p. 190.
} 


\section{Consideraciones finales}

Para terminar proponemos un conjunto de puntualizaciones de nuestro recorrido por estas consideraciones del tacto, del cuerpo propio y ajeno, con el objeto de evidenciar diferencias, continuidades y marcos comprensivos.

En primer lugar, con el enfoque desarrollado en la obra de Husserl, abordamos parcialmente su elaboración sobre la corporalidad desde la esfera del ego y el cuerpo propio, donde describe manifestaciones co-constituyentes, destacando aquellas que permiten dar cuenta de la esfera primordial. En este marco, indagamos por un lado, en sus consideraciones sobre el tacto y por otro, en la constitución del mundo objetivo a partir de la empatía entre el cuerpo propio y el ajeno. Con estas puntualizaciones hemos puesto de manifiesto aspectos subjetivistas, jerárquicos y ambiguos en su enfoque, los que entendemos mayormente ligados al horizonte de cientificidad al que se dirigen sus análisis, a sus imperativos respectivos y a la herencia comprensiva y conceptual de la metafísica tradicional. Sin embargo, las especificaciones relativas a las limitaciones en la accesibilidad al propio cuerpo, implicadas en su constitución material imperfecta, junto al énfasis puesto en las co-implicancias en la constitución del mundo objetivo, nos permiten distinguir elementos que, por un lado, modalizan el alcance del conocimiento de sí implicado en la originariedad del cuerpo propio (especialmente ligado a la imposibilidad de aprehensión definitiva ) y, por otro, revisten de ambigüedad la supuesta frontera nítida entre la plenitud intuitiva e inmediata en el conocimiento del cuerpo propio y el carácter parcial/mediato del conocimiento del ego y cuerpo del otro.

En segundo lugar, abordamos determinadas especificaciones de la corporeidad en un conjunto de seminarios de Heidegger, las cuales entendemos como pasos fundamentales para una transición respecto de las limitaciones implicadas en el enfoque de su maestro. Aquí, las indagaciones se despliegan para dar cuenta de ámbitos estructurales co-originarios de la constitución ontológica del Dasein: el carácter entramado de lo corporal, el ser-en-el-mundo y el ser-con, que no necesariamente suponen el sentido exclusivo del tacto manual para su manifestación. En nuestra lectura pusimos énfasis en el aspecto interdependiente del corporar del cuerpo, en el marco de sus estructuras constitutivas, cuya modalización se haya coimplicada con una comprensión de ser determinada: en lugar de una representación dualista-relacional, pone de manifiesto el entramado de significación y comprensivo subyacente. Asimismo, con el reconocimiento de su afinidad con las elaboraciones de madurez de Husserl (en torno al Lebenswelt) distinguimos una posible continuidad comprensiva con los desarrollos de su maestro, aunque desde un enfoque propio que permite poner en discusión las lecturas centralistas y jerárquicas de las consideraciones tradicionales del cuerpo, especialmente en lo relativo al sentido y la experiencia háptica.

Finalmente, en el tercer apartado, realizamos una lectura e interpretación de las elaboraciones de Derrida, en el marco de su contacto fraternal con los trabajos respectivos de Nancy. Asimismo, establecimos un recorrido por sus puntualizaciones de los tratamientos del tacto, tanto en trabajos de Husserl como de Heidegger. Vimos que tales abordajes se articulan como figuras de otra comprensión del tocar, esto es, con la modalidad de un con-tacto de escritura a escritura, lo cual desarrolla con detalle en sus lecturas de las elaboraciones de Nancy. En efecto, allí distingue desplazamientos, transiciones y tensiones respecto al tratamiento tradicional. 
Estas lecturas co-implican un desarrollo práctico de su comprensión del tocar y la corporeidad, permitiéndole articular sus propias elaboraciones en torno a la mesianicidad, el don, la escritura y el suplemento.

En el marco de tal articulación singular, toma distancia de las resonancias metafísicas del vocabulario tradicional del tacto, pues el riesgo de la consideración apropiante del tocar siempre puede actualizarse, en especial, considerando el lenguaje ineludiblemente heredado de la metafísica. Por ello insiste en un tocar de escritura a escritura, siempre parcial y por rodeo, múltiple y diseminal, por medio del cual no es posible una aprehensión plena o definitiva. No obstante, vimos que esta perspectiva pone de manifiesto ciertas dificultades para poder dar cuenta de la inter-corporalidad y sus relaciones respectivas.

Asimismo, con nuestras lecturas destacamos elementos en las elaboraciones sobre la corporalidad y las cuestiones referidas al tacto en Husserl y Heidegger con puntos de confluencia con la articulación comprensiva que desarrolla Derrida, en especial, en consideración del carácter extático e interrelacional de la corporalidad, más allá de los enfoques limitados al cuerpo físico-material. A nuestro entender, estas perspectivas pueden distinguirse según sea el recorrido y el compromiso (no) tematizado con el lenguaje tradicional metafísico del que son herederas. Sin embargo, estos pensadores también reconocieron el carácter ineludible de tal recorrido, en tanto condición de posibilidad para la ampliación de los límites y fronteras de la tradición heredada.

En este sentido, sostenemos que aquellas lecturas y análisis de Derrida, especialmente de los trabajos de Heidegger, revelan un abordaje parcial circunscripto al horizonte y los requerimientos de sus propias elaboraciones. No obstante, ello se haya contemplado por Derrida, para quien el con-tacto con la escritura del otro no puede ser nunca apropiante ni plena, considerando una distancia irreductible y fronteriza que preserva a la alteridad de una reducción al campo del sujeto y la representación.

Por ello, aquellas tangentes en los textos de Heidegger y Husserl no deben leerse sino como aproximaciones, rodeos de un contacto que no pretende aprehensión definitiva. Tal con-tacto aquí no pretende plenitud o cierre, aunque tampoco supone una superficie prístina u originaria de escritura sin huellas, esto es, un cuerpo de escritura in-tacto.

\section{Referencias bibliográficas}

Adrián, J. (2013): "La actualidad de la fenomenología husserliana: superación de viejos tópicos y apertura de nuevos campos de exploración”, Eidos, 18, pp. 12-45.

Agamben, G. (2019): Creación y anarquía. La obra en la época de la religión capitalista, Bs. As, Adriana Hidalgo Ed.

Aho, K. (2005): "The missing dialogue between Heidegger and Merleau-Ponty: On the importance of the Zollikon seminars", Body and Society, 11(2), pp. 1-23.

Aristóteles (2011): De Anima, Madrid, Gredos (Trad. T. Calvo).

Bernet, R. (2004): Conscience et Existence. Perspectives phenoménologiques, Presses Universitaires de France, Paris.

Breuer, I. (2015): "El esquema corporal-conocimiento experiencial de sí mismo y (auto) constitución”, Eikasia, 66, pp. 111-28. 
Bulo-Vargas, V. (2012): "Entre naturaleza y técnica: una cuestión de tacto", Revista de Filosofia, 68, pp. 55-64.

Butierrez, L. F. (2020): "Hacia el horizonte comprensivo de la corporeidad: Una transición entre los enfoques de Husserl y Heidegger". Agora. Papeles de filosofia, 79(2), pp.79106.

Butierrez, L. F. (2021): "La promesa de un cuerpo. Relaciones prácticas con la corporalidad en elaboraciones de Heidegger de 1959-1969". Thémata. Revista De Filosofia, n. ${ }^{\circ}$ 63, pp. 223-45, doi:10.12795/themata.2021.i63.12.

Candiotto, L. (2016): "Heidegger's ontic relatedness: Pros ti and Mitsein", Aurora, Rev. de filosofia, 28, 43, pp. 313-328.

Caneda-Lowry, S. (2018): "Una arriesgada posteridad. El riesgo como estrategia filosófica en Jacques Derrida y Jean-Luc Nancy”, Daimon, 74, pp. 21-41.

Depraz, N. (1995): Transcendance et incarnation. Le statut de l'intersubjectivité comme altérité à soi chez Husserl, Paris, Vrin.

Derrida, J. (1977): Semiología y gramatología, en Posiciones, Valencia, Pre-textos.

Derrida, J. (1985): “Geschlecht II. La mano de Heidegger", en Derrida (2017): Psyché. Invenciones del otro, Bs As., Ed. La Cebra, pp. 495-534.

Derrida, J. (2011): El tocar, Jean-Luc Nancy, Bs. As., Amorrortu.

Derrida, J. (2012a): La escritura y la diferencia, Barcelona, Anthropos.

Derrida, J. (2012b): Espectros de Marx, Madrid, Trotta.

De Waelhans, Alphonse (1963) 'The Philosophy of the Ambiguous', trans. Alden L. Fisher, Foreword to 2nd French edn of Maurice Merleau-Ponty's The Structure of Behavior. Boston, MA: Beacon Press., pp. xviii-xix.

Garrido Periñán, J. (2016): "Ser-en-el-cuerpo: tentativas para un esclarecimiento sobre cómo aparece un cuerpo. En y mundo", Daimon, 5, pp. 63-73.

Heidegger, M. [SuZ] (1927a): Sein und Zeit (Trad. cast. Ser y Tiempo, Santiago, Ed. Universitaria de Chile, 1997, Trad. J. E. Rivera; Ser y tiempo, Buenos Aires, FCE, 2000, trad. J. Gaos) (GA 2).

Heidegger, M. [GbM] (1929-1930): Die Grundbegriffe der Metaphysik. Welt - Endlichkeit - Einsamkeit (Winter semester 1929/30): ed. F. -W. von Herrmann, 2004 [T. C. : Los conceptos fundamentales de la metafisica. Mundo, finitud, soledad, t, Alianza Editorial, Madrid, 2007, Trad. J. A. Ciria Cosculluela] (GA 29/30).

Heidegger, M. [WhD] (1951-1952): was heisst Denken? [T. C. : ¿Qué significa pensar?, Ed. Nova, Buenos Aires, 1964. Trad. Haraldo Kahnemann. ] (GA 8).

Heidegger, M. [ZS] (1959-69): Zollikoner Seminare [T. C. : Seminarios de Zollikon, Barcelona, Herder, 2014, trad. : Ángel Xolocotzi Yáñez] (GA 89).

Husserl, E. [Ideen II] (1952[1913]): Ideen zur einer reinen Phänomenologie und phänomenologischen Philosophie II: Phänomenologische Untersuchungen zur Konstitution. Ed. Marly Biemel. La Haya, Netherlands: Martinus Nijhoff [T. C. : Ideas relativas a una fenomenología pura y a una filosofía fenomenológica II: Investigaciones fenomenológicas sobre la constitución, México, FCE, 2003, Trad: Zirión, Antonio] [Husserliana (Hua) 4].

Husserl, E. [AzpS] (1966[1926-8]): Analysen zur passiven Synthesis. Aus Vorlesungs- und Forschungsmanuskripten 1928-1926, M. Fleischer (Hrsg. ): Den Haag. (Hua 11).

Husserl, E. [CM] (1973[1931]): Cartesianische Meditationen und Pariser Vorträge, Ed. S. Strasser. La Haya, Netherlands: Martinus Nijhoff [T. C. : Meditaciones Cartesianas, Madrid, Ediciones Paulinas, 1979, Trad. : Mario Presas] (Hua 1). 
Husserl [DuR](1973[1907]): Ding und Raum. Vorlesungen 1907. Hrsg. von Ulrich Claesges, Boston/Londres: Martinus Nijhoff (Hua XVI).

Husserl, E. [Ideen I] (1988 [1913]): Ideen zu einer reinen Phänomenologie und phänomenologischen Philosophie I: Allgemeine Einfuhrung in die reine Phänomenologie, 2. Halbband: Ergänzende Texte, Ed. Karl Schuhmann. La Haya, Netherlands: Martinus Nijhoff, (1912--1929) [T. C. : Ideas relativas a una fenomenología pura y una filosofía fenomenológica, México, FCE, 1949, Trad. : José Gaos] (Hua 3-2).

Husserl, E. [AS] (2000[1920-1]): Aktive Synthesen: Aus der Vorlesung “Transzendentale Logik” 1920/21. Ergänzungsband zu "Analysen zur passiven Synthesis”, R. Breeur (Hrsg. ), Dordrecht. (Hua 31).

Husserl, E. [DL] (2008 [1916-37]) Die Lebenswelt. Auslegungen der vorgegebenen Welt und ihrer Konstitution. Texte aus dem Nachlass (1916-1937) Ed. Rochus Sowa. New York: Springer (Hua 39).

Inverso, H. (2014): El mundo entre paréntesis. Una arqueología de las nociones de reducción y corporalidad, Bs. As., Prometeo.

Iribarne, J. (2007): De la ética a la metafísica. En la perspectiva del pensamiento de Edmund Husserl, U. Pedagógica Nacional, San Pablo, Bogotá.

Johnson, F. (2016): "La exclusión del cuerpo en Sein und Zeit y la negación de una fenomenología del cuerpo en el pensamiento de Heidegger", Pensamiento, 72, 270, pp. 131-45.

Llevadot, L. (2013): "No hay mundo común: Jacques Derrida y la idea de comunidad", Isegoria, 49, pp. 549-66.

Merleau-Ponty, M. [PP](1993[1945]): Phénoménologie de la Perception, Paris, Gallimard[T. C. : Fenomenología de la percepción, Barcelona, Planeta-Agostini, Trad. Jem Cabanes].

Monteiro, H (2014): "Vivermos juntos. Tangencias entre Derrida e Nancy", Ágora, Papeles de filosofía, 33/1, pp. 101-21.

Muñoz, R. ; Delgadillo, J. (2018): “El cuerpo vivo y la subjetividad trascendental en la fenomenología de Edmund Husserl”, Veritas, 40, pp. 9-28.

Nancy, JL (2006): El Intruso, Bs. As., Amorrortu.

Nancy, JL (2010): Corpus, Madrid, Arena libros.

Presas, M. (1983): "La experiencia del otro", Escritos de Filosofía, 12, pp. 47-51.

Ramírez Cobián, M. (2017): "El cuerpo por sí mismo. De la fenomenología del cuerpo a la ontología del ser corporal", Open Insight, 14, pp. 49-68.

Ramos-González, J. (2014): "Lo avistado de Merleau Ponty de la mano de Husserl: mundo, percepción y carne”, Endoxa: Series Filosóficas, 34, pp. 209-30.

San Martín, J. (2010): “El contenido del cuerpo”, Investigaciones Filosóficas, 2, pp. 169-87.

Stein, E. (2005): Sobre el problema de la empatía, Madrid, Trotta.

Villanueva-Barreto, J. (2012): "Las bases corporales de la subjetividad en la fenomenología de Husserl”, Revista da Abordagem Gestáltica, XVIII (1), pp. 13-19.

Zahavi, D. (2004): "Husserl's Noema and the Internalism-Externalism Debate", Inquiry, 47 (1), pp. 42-66.

Zizek, S. (2018): “Pensamiento pos-secular? No, gracias”, en Zizek (2018), Contra la tentación populista, Bs. As. EGodot, pp. 72-9. 
